\title{
Bathyuriscus mendozanus (RUSCONI, 1945), TRILOBITES DEL CÁMBRICO MEDIO DE LA PRECORDILLERA ARGENTINA
}

\author{
Osvaldo Luis BORDONARO ${ }^{1}$ y Carlos Fabián \\ FOJO $^{l}$
}

${ }^{1}$ Departamento de Paleontología, IANIGLA-CCT (CONICET), CC 131, 5500, Mendoza, Argentina. obordona@mendoza-conicet.gov.ar

Bordonaro, O. L. \& Fojo, C. F. 2011. Bathyuriscus mendozanus (Rusconi, 1945), trilobites del Cámbrico medio de la Precordillera Argentina. [Bathyuriscus mendozanus (Rusconi, 1945), middle Cambrian trilobites from the Argentine Precordillera.] Revista Española de Paleontología, 26 (1), 11-23. ISSN 0213-6937.

\begin{abstract}
A taxonomic revision of the corynexochid trilobite Plesioparabolina mendozana is made and is reassigned as Bathyuriscus mendozanus, a valid species differentiated from the 19 laurentian species accepted for this genus. As well, previous open nomenclature Bathyuriscus specimens and new materials collected by the authors are included. The most fossils occur commonly in the cambrian olistoliths with outer platform mudstones, hosted in pelitic ordovician sequences. The trilobites were found in Quebrada Ojos de Agua, Quebrada Los Sombreros, Cordón del Alojamiento and San Isidro localities, from the Argentine Precordillera. The biostratigraphic distribution of the B. mendozanus is middle Cambrian, Marjumian Stage, Oryctocephalus Zone in the conventional laurentian chronology, which is equivalent in the International Subcommission on Cambrian Stratigraphy (ISCS) scale to the Cambrian Series 3, upper Stage 5 of Cambrian. Its presence in western Argentina is further evidence for the very close faunal relationship between the Precordilleran Terrane and Laurentia during the Cambrian.
\end{abstract}

Keywords: Systematic, trilobites, Cambrian Series 3, Argentine Precordillera.

\section{RESUMEN}

Se realiza una revisión taxonómica del trilobite corynexochido Plesioparabolina mendozana, que es reclasificado como Bathyuriscus mendozanus, que presenta caracteres morfológicos distintivos respecto a las otras 19 especies laurénticas incluidas en este género. También se incluyen en esta especie algunos ejemplares del mismo género previamente determinados en nomenclatura abierta y, además, se incorporan nuevos especímenes coleccionados por los autores. La mayoría de los fósiles estudiados se encuentran en olistolitos cámbricos, de calizas de plataforma externa, que se hallan alojados en pelitas ordovícicas. Los trilobites estudiados proceden de las localidades ubicadas en quebrada Ojos de Agua, quebrada Los Sombreros, Cordón del Alojamiento y San Isidro, de la Precordillera Argentina. La edad de la especie estudiada se ubica en el Cámbrico medio, Marjumiense, Zona de Oryctocephalus, según el esquema cronoestratigráfico tradicional de Laurentia, lo que equivale en el esquema bioestratigráfico mundial propuesto por la International Subcommission on Cambrian Stratigraphy (ISCS) a la Series 3, a la parte superior del Piso 5 del Cámbrico.

Palabras clave: Sistemática, trilobites, Serie 3 del Cámbrico, Precordillera Argentina.

\section{INTRODUCCIÓN}

El género Bathyuriscus Meek, 1873 es un taxón de trilobites característico y considerado endémico de Laurentia, en donde se reconocen actualmente 19 especies. Sin embargo, el mismo aparece frecuentemente en la Precordillera Argentina, aunque, hasta ahora, la calidad del material no había permitido identificarlo a nivel específico.
Con el hallazgo de nuevos ejemplares muy bien preservados y articulados que provienen de San Isidro en Mendoza, se pudo reconocer detalladamente las características morfológicas diagnósticas de estos especímenes; que han permitido, tras una revisión taxonómica detallada de algunas especies clásicas de la colección Rusconi, identificarlas como una especie ya definida, que previamente se había asignado a otro género. 
En este trabajo se realiza la revisión taxonómica del holotipo de Plesioparabolina mendozana Rusconi, 1945, cuyas características morfológicas permiten reclasificarlo como Bathyuriscus mendozanus (Rusconi, 1945). De acuerdo con esta nueva clasificación se revisan las reasignaciones hechas por otros autores de dicha especie. También se analizan los ejemplares del mismo género, hallados en varias localidades de la Precordillera, previamente determinados en nomenclatura abierta y además, se incorporan los nuevos especímenes coleccionados por los autores. Así, en la actualidad la especie está representada por más de doscientos ejemplares.

Los materiales tipos revisados provienen de San Isidro en la Precordillera de Mendoza y fueron descubiertos por primera vez por el naturalista Carlos Rusconi, quien realizó en las décadas de 1940 y 1950 numerosas colecciones de estos fósiles. Otros paleontólogos que efectuaron estudios sistemáticos de estos trilobites fueron Leanza (1947) y Poulsen (1958), pero lamentablemente los ejemplares descriptos por ellos no han podido ser ubicados y las revisiones hechas en el presente trabajo se basan en los datos bibliográficos.

\section{GEOLOGÍA Y ESTRATIGRAFÍA}

La mayoría de los fósiles estudiados en este trabajo proceden de olistolitos cámbricos, compuestos por calizas (mudstones) y pelitas oscuras, que se hallan alojados en pelitas ordovícicas. Los olistolitos cámbricos de la Precordillera Argentina fueron identificados como tales por Bordonaro (1990, 2003) y Bordonaro \& Banchig (1996). Son bloques alóctonos que tienen diferentes tamaños, que van desde pocos centímetros hasta cientos de metros y en muchos de ellos se puede reconocer una considerable sucesión estratigráfica. Se hallan resedimentados en secuencias olistostrómicas pelíticas del Ordovícico Medio a Superior conocidas como Formación Empozada (sensu Bordonaro et al., 1993) en Mendoza y Formación Los Sombreros (sensu Banchig \& Bordonaro, 1994) en San Juan. Para tener un mejor conocimiento de los olistolitos y poder identificarlos fácilmente, se les dio nombres de las localidades o quebradas donde afloran, sin la intención de crear unidades litoestratigáficas formales (Bordonaro et al., 1993; Bordonaro \& Banchig, 1996; Tortello \& Bordonaro, 1997; Bordonaro, 2003).

Los trilobites estudiados en este trabajo proceden de diferentes localidades de la Precordillera Argentina, que se encuentran tanto en la Provincia de Mendoza como en la de San Juan (Fig. 1). Si bien las mismas han sido descriptas en trabajos anteriores, a continuación se da su ubicación geográfica y se hace una breve descripción estratigráfica.

OA -Ojos de Agua: Ubicada sobre la quebrada Ojos de Agua que surca el borde oriental de la Sierra del Tontal, que integra la Precordillera Occidental de San Juan. Sobre el tramo medio de la Formación Los Sombreros aflorante en los primeros contrafuertes de esa sierra, se localiza uno de los olistolitos Ojos de Agua, con una potencia de $30 \mathrm{~m}$ columnares de calizas oscuras (mudstones) y pelitas grises (Bordonaro \& Banchig, 1990) que contiene los trilobites estudiados.

LS -Los Sombreros: Ubicada sobre el río de Los Sombreros que surca el flanco oriental de la Sierra del Tontal en la Precordillera Occidental de San Juan. Allí se halla la localidad tipo de la Formación Los Sombreros (Cuerda et al., 1983). En la mitad inferior de esa unidad se reconoce uno de los olistolitos Los Sombreros, integrado por más de un centenar de metros columnares de calizas oscuras (mudstones) y lutitas negras que contiene una abundante fauna de agnóstidos y poliméridos (Banchig \& Bordonaro, 1994; Bordonaro \& Banchig, 1996).

CA -Cordón del Alojamiento: Esta serranía se encuentra distribuida en el tramo norte de la Precordillera Mendocina y está integrada por rocas calcáreas y dolomíticas atribuidas a la Formación Alojamiento (Banchig, 2006). Sobre la quebrada de Aguilera y en la parte media de dicha formación, hay una escama tectónica muy deformada que alcanza unos $100 \mathrm{~m}$ estratigráficos, compuesta por calizas lajosas (mudstones y wackestones) oscuras que son portadoras de la fauna estudiada junto a otros agnóstidos, oryctocefálidos y alokistocáridos (Bordonaro \& Banchig, 2007)

SI -San Isidro: Esta localidad se encuentra en el extremo austral de la Precordillera Mendocina, al oeste de la ciudad capital de Mendoza, en las inmediaciones de la Estancia San Isidro, donde aflora la Formación Empozada (sensu Bordonaro et al., 1993). Allí están expuestos varios olistolitos de gran tamaño, de varias decenas de metros columnares, que fueron llamados olistolitos San Isidro (Fig. 2). Es posible reconocer hacia el tope de los mismos un tramo de limolitas y margas intercaladas con lentes de calizas (mudstones, wackestones y packstones) fosilíferas formando coquinas de trilobites (Keller et al., 1993). De esos niveles provienen la mayoría de los ejemplares nuevos aquí estudiados, como también los coleccionados por Rusconi, Leanza y Poulsen. Las localidades referidas por estos últimos autores, puntualmente dentro del área de San Isidro, son enumeradas a continuación (Fig. 2): 1- "500 m al oeste de la Estancia San Isidro" (Rusconi, 1945), que también es conocida como yacimiento clásico. 2- "Sobre la quebrada de San Isidro a unos $500 \mathrm{~m}$ aguas arriba de la estancia del mismo nombre" (Leanza, 1947), que también corresponde al llamado yacimiento clásico. 3- "cerro Martillo" (Poulsen, 1958), ubicada a unos $1.500 \mathrm{~m}$ al norte de la Estancia San Isidro. Con respecto a los ejemplares nuevos coleccionados por los autores, proceden de las siguientes localidades (Fig. 2): 4- quebrada San Isidro (yacimiento clásico), 5- quebrada Empozada, 6- filo La Cruz, 7- quebrada Oblicua y 8- cerro Martillo. 


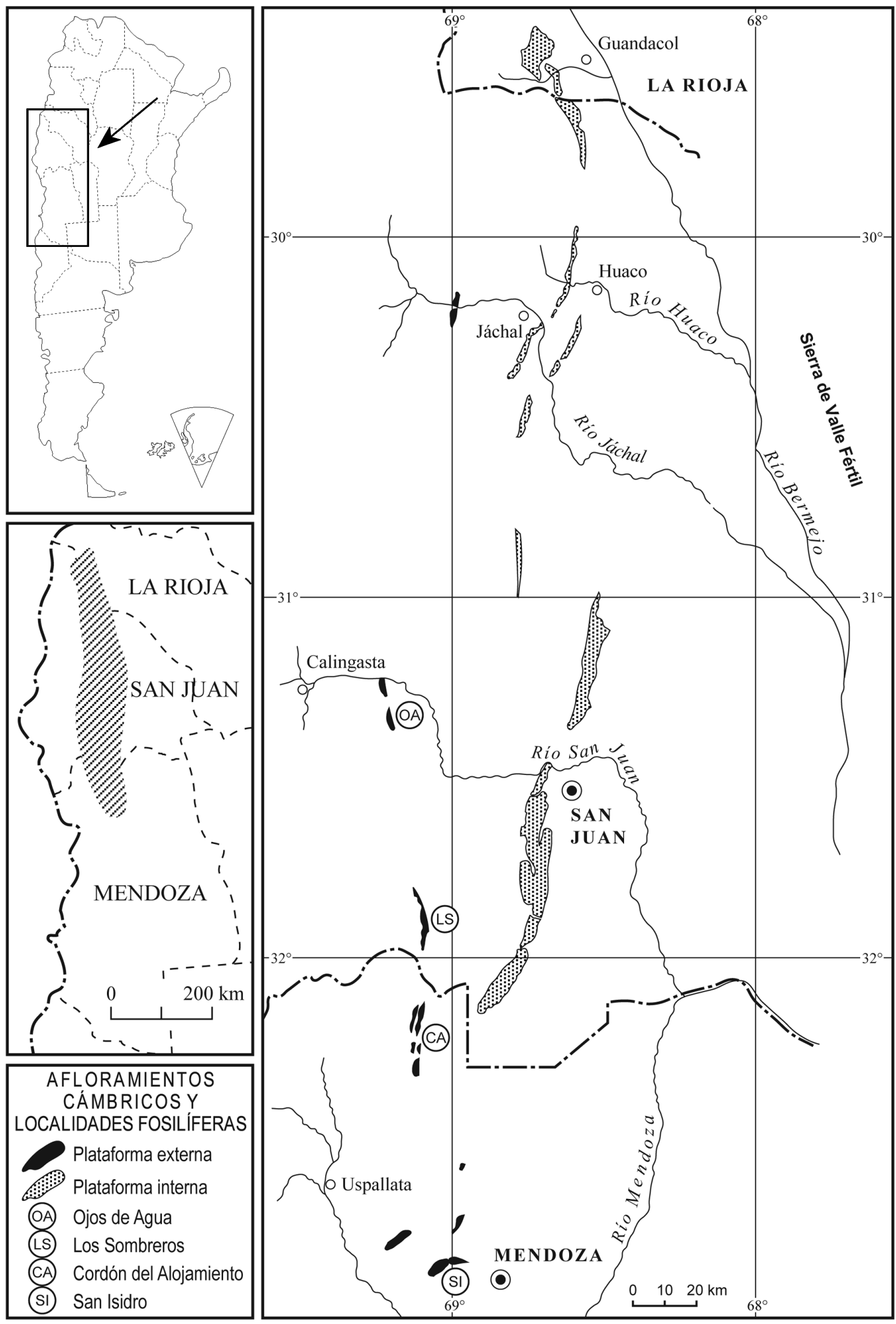

Figura 1. Mapa de ubicación de afloramientos cámbricos y de localidades fosilíferas donde aparece Bathyuriscus mendozanus (Rusconi, 1945) en la Precordillera de San Juan y Mendoza, Argentina.

Geological map with cambrian outcrops and Bathyuriscus mendozanus (Rusconi, 1945) localities in the Precordillera of Mendoza and San Juan, Argentina. 


\section{MATERIALES Y MÉTODOS}

El material utilizado para este trabajo se basa en ejemplares de la colección de trilobites de Rusconi depositada en el Museo de Ciencias Naturales y Antropológicas "Juan Cornelio Moyano" de la Provincia de Mendoza, que figura con la sigla MCNAM-PI. Los ejemplares citados por Leanza, que fueron depositados en el Museo de la Dirección General de Minas y Geología (Buenos Aires), actual SEGEMAR (Servicio Geológico Minero Argentino) no han sido hallados y por lo tanto se los considera extraviados. Los ejemplares coleccionados por Poulsen no pudieron ser ubicados ya que no están registrados en ningún repositorio. Los especímenes previamente determinados en nomenclatura abierta que proceden de la Precordillera Occidental de San Juan, se hallan depositados en el Repositorio de Paleontología de Invertebrados de la Facultad de Ciencias Exactas, Físicas y Naturales de la Universidad Nacional de
San Juan, con la sigla PIUNSJ. En cambio, los que proceden de la Precordillera mendocina se hallan depositados en el repositorio de invertebrados fósiles del Instituto Argentino de Nivología, Glaciología y Ciencias Ambientales que funciona en el Centro Científico Tecnológico de Mendoza, con la sigla IANIGLA-PI. Como materiales complementarios se han usado colecciones realizadas por los autores, las que también se hallan en el repositorio de invertebrados fósiles del Instituto Argentino de Nivología, Glaciología y Ciencias Ambientales (sigla IANIGLA-PI). Este nuevo material fue hallado en las localidades indicadas en el capítulo anterior (Fig. 2) y todos provienen de los tramos cuspidales de los denominados olistolitos San Isidro. Estos ejemplares nuevos se han utilizado para comprobar y ampliar las características morfológicas de cada uno de los integrantes de esta especie.

Estos estudios se basan en el empleo de criterios morfológicos registrados en los caparazones y su comparación

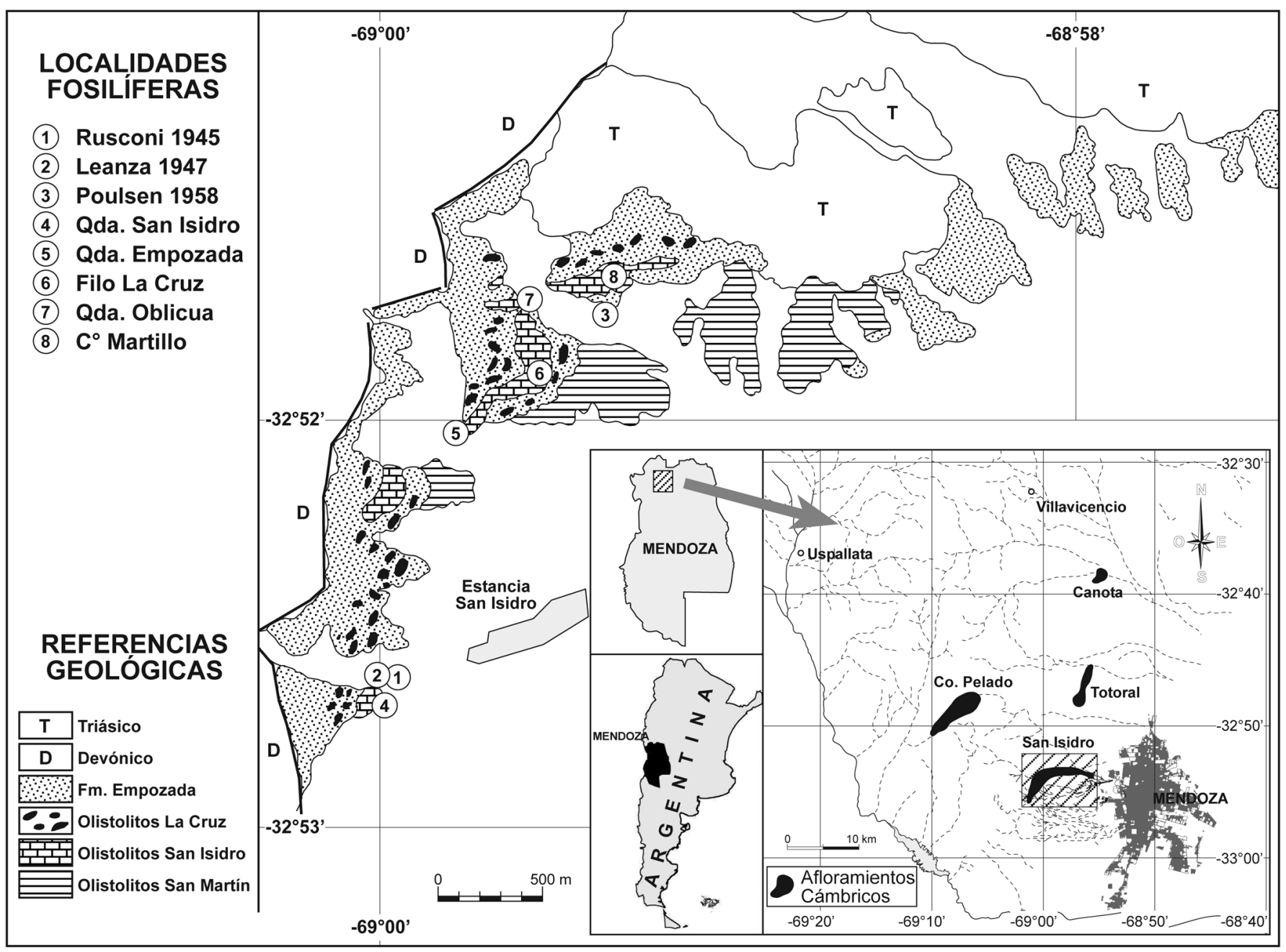

Figura 2. Mapa de ubicación y de afloramientos cámbricos de la localidad de San Isidro, Precordillera de Mendoza, Argentina. 1-8: Ubicación de localidades fosilíferas citadas en el texto.

Geological map with cambrian outcrops and fossil localities (1-8) cited in the text, in San Isidro Area, Precordillera of Mendoza, Argentina. 
con otras formas citadas en la bibliografía mundial. Todos los especímenes estudiados fueron limpiados con medios de percusión y vibración, blanqueados con óxido de magnesio y fotografiados con una cámara digital Olympus incorporada a un microscopio binocular Olympus SZ61.

\section{SISTEMÁTICA}

Clase TRILOBITA Walch, 1771

Orden CORYNEXOCHIDA Kobayashi, 1935

Familia Dolichometopidae Walcott, 1916

\section{Género Bathyuriscus Meek, 1873}

Especie tipo. Bathyurus (?) haydeni Meek, 1873.

Diagnosis enmendada: Género de Dolichometopidae con cranidio subtrapezoidal. Glabela de lados paralelos o anteriormente expandidos donde el $1^{\circ}, 2^{\circ}$ y $4^{\circ}$ surcos glabelares son someros y angostos mientras que el $3^{\circ}$ es somero o ausente. Lóbulo palpebral semicircular cuyo largo es $1 / 3$ a 1/2 del largo cranidial. Mejilla fija posterior ancha y corta. Tórax compuesto por ocho a doce segmentos. Pigidio grande de contorno suboval o semicircular con surcos pleurales largos y surcos interpleurales cortos pero distintivos y con borde elevado y angosto.

A genus of Dolichometopidae with subtrapezoidal cranidium. Glabella parallel-sided or anteriorly expanding. $1^{\circ}, 2^{\circ}$, and $4^{\circ}$ furrows shallow and narrow. $3^{\circ}$ furrow shallow or absent. Palpebral lobe crescentic, about one third to one half of the cranidial length. Posterior fixed cheek wide and short. Thorax composed of eight to twelve segments. Pygidium large, subovate or semicircular in outline. Pleural furrows long, interpleural furrows short but distinct. Pygidial border narrow and raised.

Discusión: Robison (1964) consideró Bathyuriscus, Orria Walcott, 1916 y Orriella Rasetti, 1948 co-genéricos y relacionados por los siguientes rasgos: una glabela de lados paralelos o también anteriormente expandida, 9 segmentos torácicos, pigidio macropigo con un borde angosto y elevado, distintivos surcos pleurales e interpleurales. Reconoció sólo 9 especies como pertenecientes a este género. Posteriormente, Robison (1967) hizo un detallado estudio ontogenético de Bathyuriscus fimbriatus Robison, 1964 donde destacó que todos los tórax del estadio holáspido tenían 9 segmentos y espina media axial. Robison (1976) sugirió que Bathyuriscus podría ser un sinónimo antiguo de Wenkchemnia Rasetti, 1951, ya que ambos parecen representar los extremos de una línea evolutiva continua y sólo se diferencian en el número de segmentos torácicos y tamaño del pigidio. Young \& Ludvigsen (1989) ampliaron la diagnosis de Bathyuriscus destacando algunos detalles en el cranidio y el pigidio. En esa diagnosis, estos últimos autores no dijeron nada de las características del tórax. Más aún, crearon 3 nuevas especies utilizando sólo los caracteres de cranidios y pigidios, e incorporaron 3 especies más de Bathyuriscus a las 9 aceptadas por Robison (1964). Babcock (1994) anexó a la diagnosis de Bathyuriscus la presencia de sólo un par de fosetas en el surco axial, en lugar de dos. También coincidió en que Wenkchemnia es un joven sinónimo de Bathyuriscus. Wolfart (1994) destacó algunos detalles morfológicos de Bathyuriscus tanto en el cranidio como en el pigidio, pero no modificó la diagnosis del género. Sundberg (1994) reivindicó a Wenkchemnia, como género válido por tener un pigidio más pequeño y por no tener surco en el borde pigidial, como también señaló que la profundidad de los surcos interpleurales y axiales es tan variable en los Corynexochidos que por sí mismos no son garantía de la separación de dos géneros.

En lo referido al número de segmentos torácicos se puede destacar que Walcott (1916) describió Bathyuriscus (Poliella) powersi con 11 segmentos torácicos con nodos o espinas en todos ellos. Sin embargo Robison (1964) los excluyó del género Bathyuriscus por no tener 9 segmentos torácicos aunque ahora es aceptada como una especie del género. También McLaughlin \& Enbysk (1950) ilustraron y describieron un Bathyuriscus sp. con 10 segmentos torácicos. Otros autores reconocieron especies de Bathyuriscus sin la presencia de los tórax (Rasetti, 1967; Fritz, 1968; Palmer, 1968; Kindle, 1982; Babcock, 1994). Robison (1971: lámina 90, figura14) ilustró un especimen de B. fimbriatus con 8 segmentos torácicos en lugar de los 9 antes diagnosticado. Sobre esta temática hay que mencionar que Hughes (1994) en un estudio sobre la variabilidad intraespecífica de Dikelocephalus Owen, 1852 destacó que la variación en el número de segmentos torácicos en las especies de trilobites es un hecho común. También Elrathia kingii (Meek, 1870) muestra estados adultos con 10 a 13 segmentos torácicos (Bright, 1959) y Paradoxides davidis brevispinus Bergström \& Levi-Setti, 1978 contiene adultos con 19 a 21 segmentos torácicos (Bergstrom \& Levi-Setti, 1978).

Actualmente se aceptan 19 especies confirmadas de Bathyuriscus (M. Webster comunicación personal) entre las cuales está incluida B. powersi Walcott, 1916 que posee 11 segmentos torácicos, otras 9 especies con nomenclatura abierta que no tienen los tórax y Bathyuriscus sp. de McLaughlin \& Embysk (1950) que tiene 9 o 10 segmentos torácicos.

La discusión precedente sirve de argumento para demostrar que el número de segmentos torácicos por sí solo no debe ser considerado como una característica morfológica diagnóstica en los géneros de trilobites poliméridos cámbricos, como es el caso de Bathyuriscus. En consecuencia, el número de segmentos torácicos de Bathyuriscus no puede limitarse a 9 sino que debe aceptarse una variabilidad entre 8 y 12 , tal como se propone en la diagnosis enmendada. 
Biogeografía: Bathyuriscus es un género endémico de Laurentia que se encuentra distribuido en casi toda la periferia del cratón norteamericano. La presencia de este género en la Precordillera del oeste de Argentina denota una cercana relación biogeográfica entre Laurentia y el terreno exótico de Cuyania del cual forma parte la Precordillera (Ramos, 2004). En la actualidad, la aparición de Bathyuriscus sólo se registra en las facies de plataforma externa de la cuenca cámbrica de la Precordillera Argentina y hasta el momento no ha sido hallado en la plataforma interna (Bordonaro et al., 2008).

\section{Bathyuriscus mendozanus (Rusconi, 1945)} Figuras 4 y 5

* v 1945. Plesioparabolina mendozana Rusconi: 216, fig. 1.

p 1947. Amecephalus mendozanus (Rusconi), Leanza: 232 (sólo la sinonimia, no lámina 1 , fig. 13)

1950. Amecephalus mendozaensis (Rusconi), Rusconi: 162.

p 1958. Kistocare mendozanum (Rusconi), Poulsen: 14 [sólo parte de la sinonimia, no Amecephalus mendozanus (Rusconi) Leanza ni Syspacephalus asperoensis Rusconi].

p 1963. Kistocare mendozanum (Rusconi), Castellaro: 19, fig. 1 (sólo el tórax y el pigidio, no el cranidio).

v 1990. Bathyuriscus aff. rotundatus, Bordonaro \& Banchig: 32, lám.1, figs. 1 y 2.

v 1990. Bathyuriscus sp. indet., Bordonaro \& Banchig: 32, Lám. 1, figs. 3 y 4.

v 1995. Parkaspis endecamera, Bordonaro \& Banchig: lám. 2, fig. 6.

v 1996. Bathyuriscus sp., Bordonaro \& Banchig: 475.

v 2007. Bathyuriscus sp. indet., Bordonaro \& Banchig: 99, fig. 2. F-H.

Materiales: Se examinaron los materiales originales de Plesioparabolina mendozana Rusconi, 1945 (holotipo MCNAM-PI 2091 y materiales adicionales MCNAM-PI 2086 a 2159 y 13885 al 13888, 14544 al 14555 y 18464) de la colección Rusconi procedente de San Isidro, Mendoza (Museo de Ciencias Naturales y Antropológicas "Juan Cornelio Moyano"). Los nuevos ejemplares recolectados por los autores en los olistolitos de San Isidro (IANIGLA-PI: 2072 al 2140, 2249, 2275 al 2320), lo integran 80 cranidios, 36 pigidios, 55 tórax y 3 mejillas libres. Además, se dispone de 8 pigidios, 3 cranidios y 4 librígenas provenientes del Cordón del Alojamiento (Fm. Alojamiento) de Mendoza (IANIGLA-PI 1574 a 1588); de 8 cranidios y 4 pigidios de los olistolitos Los Sombreros (IANIGLA-PI 2341 a 2350) de la Fm. Los Sombreros aflorante en la Sierra del Tontal, San Juan, y de 2 pigidios y 1 cranidio de los olistolitos Ojos de Agua (PIUNSJ 516 a 518) de la Fm. Los Sombreros aflorante en la Sierra del Tontal, San Juan.

Descripción original: Rusconi (1945) basó su descripción en un especimen casi completo, considerado como holotipo, y en varios especímenes fragmentados, destacando la siguiente morfología: Glabela relativamente alargada con tres pares de surcos laterales no marcados en la zona axial, siendo los posteriores menos acentuados. Espinas genales relativamente prolongadas llegando casi hasta la mitad de la longitud del tórax. Tórax con 12 a 13 segmentos (cabe aclarar que el holotipo posee sólo 12 y no se ha hallado ningún ejemplar con 13), con faceta articular bien definida. Las pleuras tienen surco central ancho y terminan casi a las nacientes de las espinas, las espinas cortas y dirigidas oblicuamente hacia afuera y hacia atrás. Pigidio con 4 o 5 segmentos, siendo el último anillo muy reducido. Borde posterior semiesférico con surco marginal donde terminan los segmentos axiales y respectivos surcos. Raquis adelgazado hacia atrás.

Poulsen (1958) anexó a esta descripción la presencia de un cuarto par anterior de surcos glabelares bien marcados y convergentes suavemente hacia adelante.

Descripción actualizada: Céfalo de contorno semicircular. Glabela elongada tocando el borde anterior, expandida hacia adelante a partir del segundo par de surcos glabelares, con 4 pares de surcos glabelares: $1^{\circ}$ ancho, profundo y dirigido hacia atrás, $2^{\circ}$ corto, profundo y recto, $3^{\circ}$ y $4^{\circ}$ angostos, someros y dirigidos hacia adelante. Anillo occipital ancho y con una diferenciada espina occipital dirigida hacia atrás. Borde anterior muy angosto y levantado, plataforma anterior de las fixígenas cóncavas y relativamente angostas. Áreas palpebrales de las fixígenas cuyo ancho es la mitad del ancho glabelar en su punto medio. Lóbulos palpebrales largos y uniformemente curvados, casi tocan la glabela entre el $3^{\circ}$ y $4^{\circ}$ surco glabelar. Áreas posteriores de las fixígenas largas y anchas. Surco marginal posterior recto, ancho y somero distalmente, angosto y profundo axialmente. Sutura facial divergente por delante de los ojos convergiendo en el borde anterior. Mejillas libres anchas y planas con espina genal delgada y larga llegando casi al quinto anillo torácico. Tórax compuesto por 10 a 12 segmentos torácicos. Axis prominente con espinas o nodos axiales en el centro de todos los anillos axiales. Pleuras que terminan en cortas y delgadas espinas pleurales curvadas levemente hacia atrás, con surcos pleurales anchos y someros adelgazándose distalmente. Abultamiento triangular dentro del surco pleural adyacente al axis de moderada prominencia y cuyo ancho es alrededor de 1/4 del ancho total de la pleura. Pigidio semicircular con axis que posee entre 4 y 5 anillos axiales más una pieza terminal, suavemente adelgazado en el extremo terminal que no alcanza a tocar el borde marginal. En algunos ejemplares se observa una difusa cresta postaxial extendida desde la pieza terminal hasta tocar el borde marginal. Borde marginal uniforme, angosto, poco elevado y sin espinas. La superficie de los caparazones es suave y sin pústulas. En la figura 3 se puede apreciar una reconstrucción gráfica de un ejemplar ideal y completo de B. mendozanus.

Discusión: Plesioparabolina mendozana Rusconi, 1945 se basó en el holotipo MCNAM-PI 2091 que no fue fotografiado y en otros 73 ejemplares adicionales colectados en San Isidro, Mendoza (localidad 1 de la figura 2). El dibujo del holotipo realizado por su autor (Rusconi, 1945: 218, figura 1) lo muestra como un ejemplar articulado con el tórax y pigidio completos, pero el céfalo está incompleto. Rusconi la clasificó erróneamente en el Orden Ptychopariida, Familia Olenidae y género Plesioparabolina Harrington \& Leanza, 1942. La observación detallada del holotipo (Fig. 4.k), permite apreciar una glabela alargada $\mathrm{y}$ con surcos axiales paralelos, un tórax y un pigidio con morfologías típicas del Orden Corynexochida, de la Fami- 


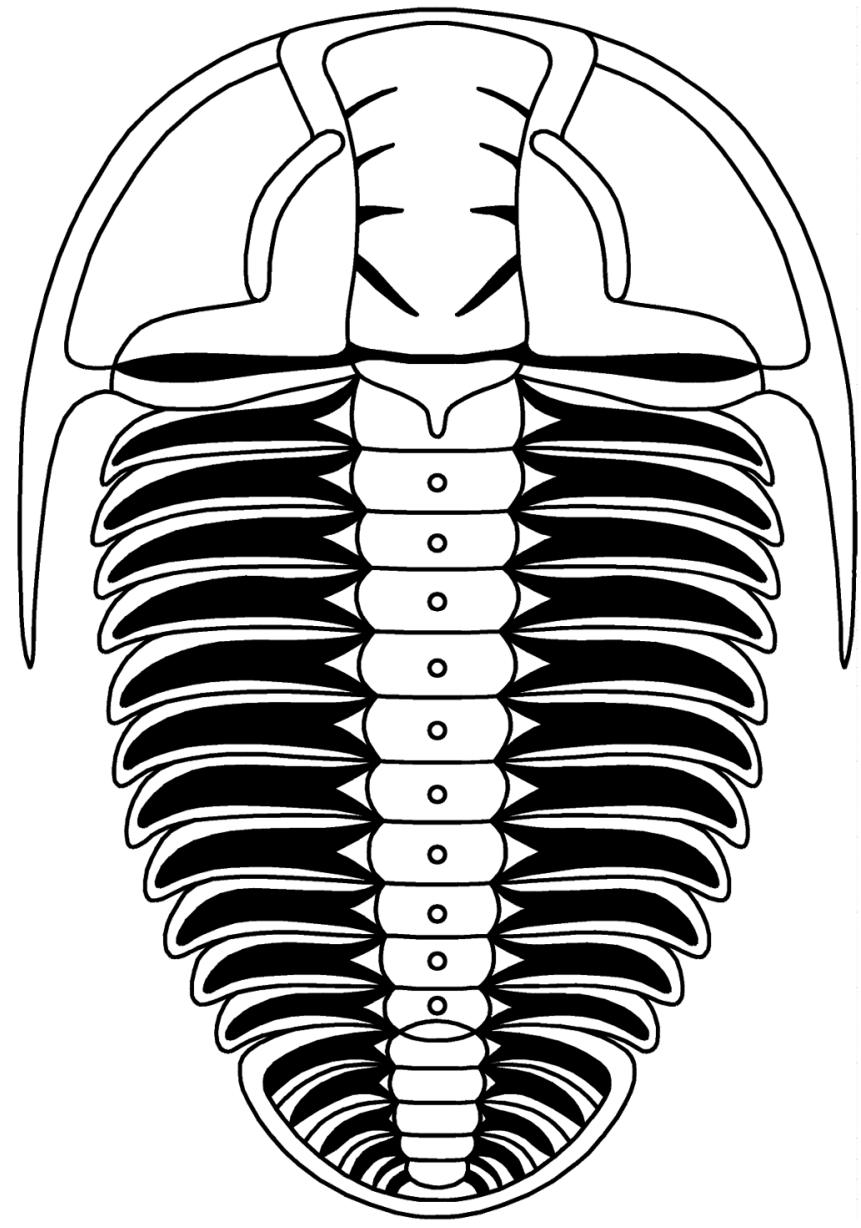

Figura 3. Reconstrucción gráfica de Bathyuriscus mendozanus (Rusconi, 1945).

Graphic reconstruction of Bathyuriscus mendozanus (Rusconi, 1945).

lia Dolichometopidae y del género Bathyuriscus tal como se discutió en el apartado sobre el género.

De esta manera, se considera que los especímenes estudiados en este trabajo poseen los rasgos diagnósticos del género Bathyuriscus: Tienen la glabela anteriormente expandida y con 4 pares de surcos laterales someros $\left(1^{\circ}\right.$ y $2^{\circ}$ bien diferenciados, $3^{\circ}$ y $4^{\circ}$ someros) (ver Figs. 4.a-f, 5.f-i). Los lóbulos palpebrales son de 1/3 a 1/2 del largo cranidial y están situados ligeramente por detrás del punto medio de la glabela (ver Fig. 4.a-f). El tórax está compuesto por 10 a 12 segmentos (ver Fig. 4.a-c y 4.k). El pigidio es grande, de contorno semicircular, con surcos pleurales e interpleurales bien marcados y con un borde angosto bien definido y elevado que está limitado por un distintivo surco (ver Figs. 4.g-k, 5.j). Otra característica que poseen los especímenes precordilleranos es el abultamiento triangular dentro del surco pleural adyacente al axis que, según Robison (1964), es característico del género Bathyuriscus. También es notable el surco pleural ancho y somero adelgazándose levemente hacia el extremo dis- tal y las bandas pleurales anteriores y posteriores de cada segmento pleural que mantienen un diseño paralelo entre ellas a lo largo de todo su recorrido en la pleura (ver Figs. 4.b, 4.h, 4.i, 5.b, 5.j).

Los caracteres distintivos de Bathyuriscus mendozanus que indican que es una especie válida, son la presencia de una importante espina occipital dirigida hacia atrás, áreas palpebrales de las fixígenas cuyo ancho es la mitad del ancho glabelar en su punto medio, mejillas fijas posteriores anchas. Tórax con 10 a 12 segmentos torácicos con axis prominente y con espinas o nodos axiales en el centro de todos los anillos axiales. Pigidio semicircular cuyo ancho es el doble del largo, con axis que casi toca el borde marginal, que posee de 4 a 5 anillos axiales más una pieza terminal; surco del borde marginal bien definido, borde marginal uniforme, angosto y sin espinas.

Aunque no se han realizado estudios morfométricos en los especímenes de $B$. mendozanus, ellos muestran un amplio rango de variaciones morfológicas que, a nuestro entender, son superfluas y no tienen importancia específica, y que pueden considerarse como parte de la variabilidad intraespecífica o bien, estar vinculadas a condiciones de preservación y diagénesis (ver Figs. 4 y 5). Así, es común observar como varía el ancho del borde anterior del cranidio, la curvatura de los lóbulos palpebrales, la profundidad o angularidad de los surcos glabelares laterales, la curvatura de los surcos glabelares axiales, la mayor o menor curvatura en el borde anterior de la glabela y hasta la espina occipital que, a veces, es conspicua y otras veces es casi imperceptible o está insinuada por un tubérculo. Los ejemplares de la colección Rusconi, como los de la colección propia, muestran un número variable de segmentos torácicos que va desde 10 hasta 12 y son generalmente ejemplares completos o casi completos donde el tórax está frecuentemente presente y articulado tanto con el pigidio como con el cranidio. Así, el holotipo MCNAMPI 2091 de $B$. mendozanus, posee 12 segmentos torácicos (Fig. 4.k), mientras que los ejemplares IANIGLA-PI 2089, 2086, 2085 tienen 10 (Figs. 4.a, 4.c) y los ejemplares IANIGLA-PI. 2087, 2099 tienen 11 (Fig. 4.b). También la presencia del tubérculo medio en los anillos axiales puede ser muy notable o levemente insinuada (ver Figs. 4.a, 4.e, 4.g). La cantidad de anillos axiales en el pigidio varía entre 4 y 5 (ver Figs. 4.g, 4.i), la distancia de la pieza terminal hasta el borde pigidial, a veces, casi lo toca y otras veces se encuentra más distante (ver Figs. 4.h, 4.k) o hasta tiene una pequeña cresta post-axial que la conecta con el borde pigidial (ver Fig. 4.j).

La especie más cercana a $B$. mendozanus es $B$. rotundatus (Rominger, 1887) Walcott (1916: lámina 47, figuras 2, 2a, 2b) de la Formación Stephen, en las Rocallosas de Canadá (Rasetti, 1951). Se parecen principalmente en la forma y tamaño del pigidio, donde ambas especies tienen 4 a 5 segmentos axiales más una pieza terminal subtriangular que casi toca el borde marginal que es angosto, ele- 

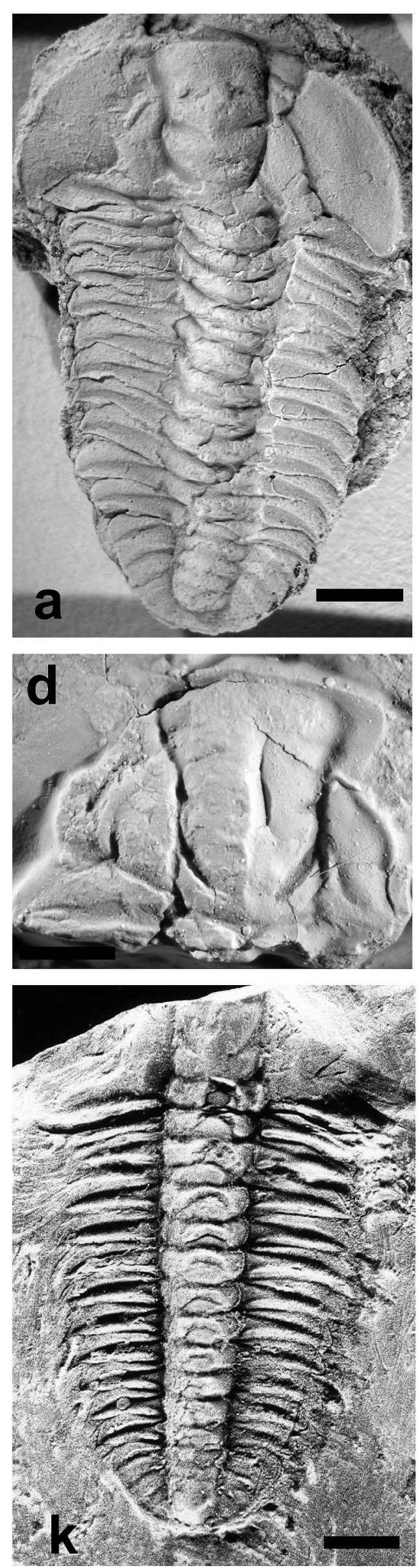
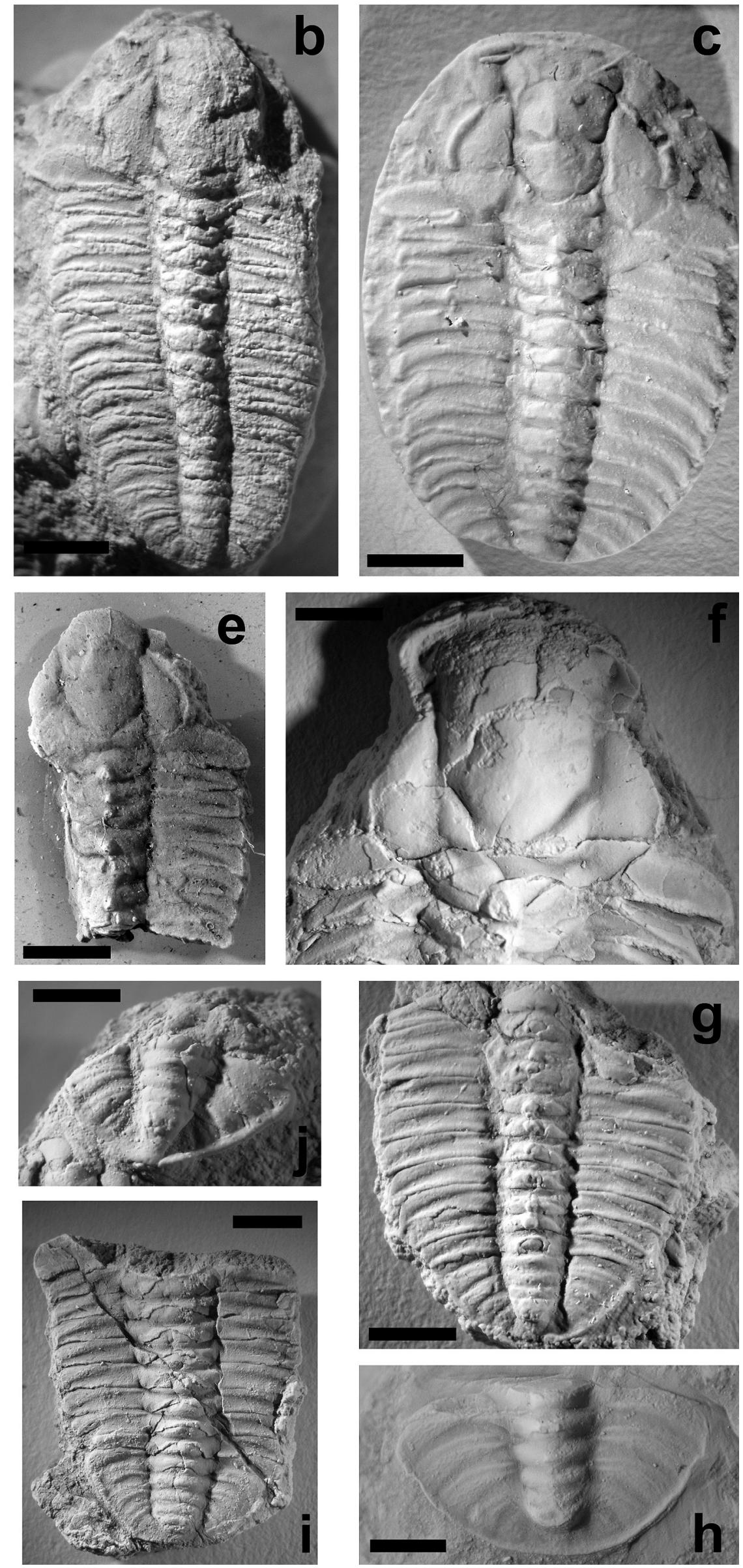
vado y sin espinas. También son similares los surcos pleurales bien marcados, anchos y profundos, que se extienden en forma paralela a los surcos interpleurales hasta tocar el borde marginal. Las diferencias más notables entre las dos especies están en el número de segmentos axiales del tórax, que en $B$. rotundatus es de $9 \mathrm{y}$ sin espinas axiales. También en $B$. rotundatus la glabela es más expandida y globosa anteriormente y las áreas palpebrales de las mejillas fijas son más angostas.

La especie tipo B. haydeni (Meek, 1873) tiene similitud en la forma y tamaño del pigidio con 5 a 6 segmentos axiales, aunque el borde pigidial es más ancho. Se diferencia notablemente porque el tórax tiene 9 anillos torácicos y las áreas palpebrales de las mejillas fijas son más angostas.

Bathyuriscus (Poliella) balus Walcott, 1916 (lámina 49, figuras 1a-g), fue definido en base a varios ejemplares conservados en lutitas, que en general están deformados. No obstante eso, la gran cantidad de individuos permitió a Walcott (1916) hacer una buena reconstrucción de un ejemplar completo como lo dibuja en la figura 1 de la lámina. Entre los caracteres diagnósticos que comparte con B. mendozanus se destacan claramente 10 segmentos torácicos todos con prominentes espinas axiales medias, el pigidio semicircular con 4 anillos axiales más una pieza terminal y un bien definido borde marginal; los lóbulos palpebrales uniformemente curvados y elongados, las áreas palpebrales tan anchas como la mitad del ancho glabelar y un anillo occipital ancho con prominente espina occipital. Las diferencias más notables con la especie precordillerana son la forma de la glabela que no está expandida anteriormente y las mejillas libres que son más robustas y con espinas genales cortas y gruesas.

Bathyuriscus powersi Walcott (1916: lámina 46, figura 1), es un único ejemplar completo donde se aprecia un pigidio pequeño semicircular con 3 anillos axiales, más una pieza terminal y borde angosto, el tórax con 11 segmentos con nodo axial en todos ellos e incluso en el anillo occipital. Las diferencias con B. mendozanus se manifiestan en el cranidio que tiene más angostas las mejillas fijas y los lóbulos palpebrales que no son tan curvados sino rectos y dirigidos hacia atrás. También difiere en el pigidio que es mucho más pequeño.
Amecephalus mendozanus (Rusconi, 1945) Leanza (1947) fue reclasificado sobre la sinonimia de Plesioparabolina mendozana, como también se incluyó en esta especie a otro ejemplar proveniente de San Isidro, Mendoza (localidad 2, Fig. 2), y aunque el ejemplar no ha sido visto por hallarse extraviado, pertenece sin dudas al Orden Ptychopariida y a la Familia Alokistocaridae (ver Leanza, 1947: 273, lámina I, figura13). Por ello la sinonimia aquí indicada es parcial y sólo referida a Plesioparabolina mendozana.

Amecephalus mendozaensis (Rusconi, 1945) es la reasignación de Plesioparabolina mendozana que hace Rusconi (1950: 162) siguiendo la denominación hecha por Leanza (1947).

Bathyuriscus sp. de McLaughlin \& Enbysk (1950: lámina 65 , figura 4) se parece por la forma y características del cranidio, el número de segmentos torácicos y el tamaño y forma del pigidio. No obstante, el único ejemplar fotografiado está muy deformado y se hace difícil establecer la especie a la que pertenece hasta no disponer de nuevos materiales.

Wenkchemnia spinicollis Rasetti, 1951 (op. cit: : figura 11, 4-8) tiene muchas similitudes con B. mendozanus en el cranidio, pero difiere en el pigidio al tener un borde pigidial muy difuso y plano y carecer del surco del borde, como sí los tiene $B$. mendozanus.

Kistocare mendozanum (Rusconi, 1945) Poulsen (1958) fue redefinido en base a Plesioparabolina mendozana, a Amecephalus mendozanus según Leanza (1947), a Syspacephalus asperoensis Rusconi, 1952 y a ejemplares nuevos procedentes del cerro Martillo, en San Isidro, Mendoza (localidad 5, Fig. 2), que no fueron ilustrados. De los tres integrantes de la sinonimia original hecha por Poulsen, solamente se considera a Plesioparabolina mendozana como sinónimo de $B$. mendozanus.

En el dibujo realizado por Castellaro (1963: 19, figura 1) de Kistocare mendozanum (Rusconi, 1945), se aprecia que el céfalo es de un alokistocarido, en cambio el tórax y pigidio es típico de un dolichometopido. Por lo tanto la sinonimia de ese ejemplar con Bathyuriscus mendozanus es parcial y sólo referida al tórax y pigidio.

Bathyuriscus sp. de Rasetti (1967: lámina12, figs. 2931 ), aunque se parece a $B$. mendozanus por la forma ge-

Figura 4. a-k, Bathyuriscus mendozanus (Rusconi, 1945) de los olistolitos San Isidro, Formación Empozada, Precordillera de Mendoza; a, caparazón completo IANIGLA-PI 2085. b, caparazón incompleto IANIGLA-PI 2087. c, caparazón incompleto IANIGLA-PI 2089. d, cranidio IANIGLA-PI 2129. e, cranidio y tórax incompletos IANIGLA-PI 2076. f, cranidio IANIGLA-PI 2138. g, tórax incompleto y pigidio IANIGLA-PI 2088. h, pigidio IANIGLA-PI 2096. i, tórax incompleto y pigidio IANIGLA-PI 2094. j, pigidio IANIGLA-PI 2091. k, caparazón incompleto de Plesioparabolina mendozana Rusconi, 1945, holotipo MCNAM-PI 2091. Escala gráfica $=2,5 \mathrm{~mm}$.

$\boldsymbol{a}-\boldsymbol{k}$, Bathyuriscus mendozanus (Rusconi, 1945) from the San Isidro olistoliths, Empozada Formation, Precordillera of the Mendoza; $\boldsymbol{a}$, complete carapace IANIGLA-PI 2085. b, incomplete carapace IANIGLA-PI 2087. c, incomplete carapace IANIGLA-PI 2089. d, cranidium IANIGLA-PI 2129. e, cranidium and incomplete thorax IANIGLA-PI 2076. $\boldsymbol{f}$, cranidium IANIGLA-PI 2138. $\mathbf{g}$, incomplete thorax and pygidium IANIGLA-PI 2088. $\boldsymbol{h}$, pygidium IANIGLA-PI 2096. $\boldsymbol{i}$, incomplete thorax and pygidium IANIGLA-PI 2094. $\boldsymbol{j}$, pygidium IANIGLA-PI 2091. $\boldsymbol{k}$, incomplete carapace of the Plesioparabolina mendozana Rusconi, 1945, holotype MCNAM-PI 2091. Bar scale $=2.5 \mathrm{~mm}$. 

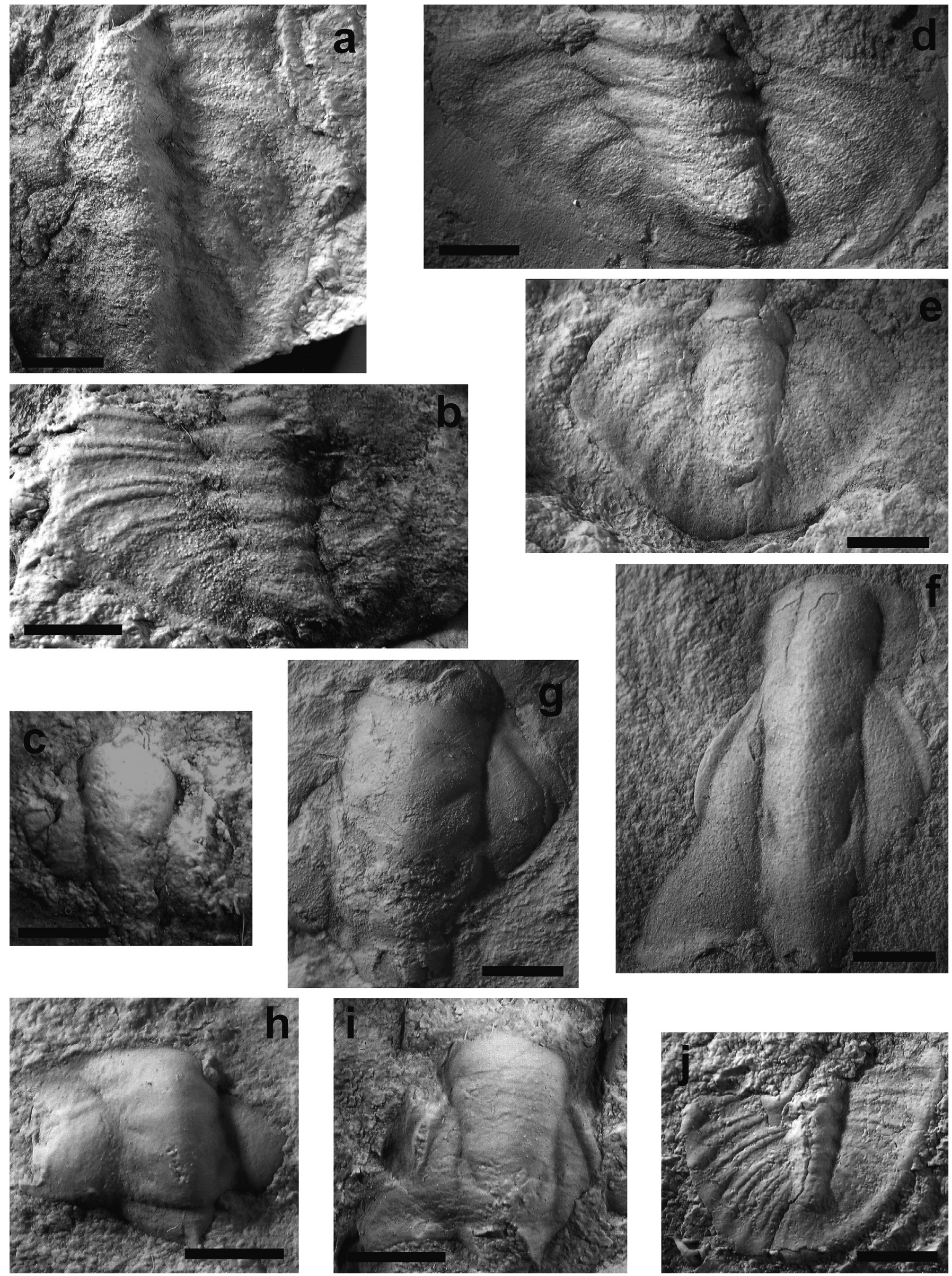
neral y tamaño del pigidio, difiere en que es más alargado transversalmente, tiene más pronunciados los surcos interpleurales y posee una leve muesca en la parte axial del borde marginal.

Bathyuriscus sp. de Kindle (1982: lámina 1.1, figura 21) comparte el tamaño, la forma y proporciones del único pigidio ilustrado, con 5 anillos axiales más una pieza terminal que casi toca el borde marginal. Se diferencia en que tiene una corta cresta postaxial que une la pieza terminal con el borde pigidial y además, tiene una pequeña muesca del borde pigidial en la zona axial. Más tarde, este pigidio fue incluido en la sinonimia de Bathyuriscus boscaputensis Young \& Ludvigsen (1989: 15).

Bathyuriscus boscaputensis Young \& Ludvigsen, 1989, de Terranova occidental, se basa en 13 cranidios y 20 pigidios no articulados y sin tórax. Los pigidios de esa especie canadiense se parecen a $B$. mendozanus porque son subovales, más anchos que largos y tienen 4 anillos más la pieza terminal con una pequeña cresta post-axial que la conecta con el borde pigidial, características que también se suelen ver en algunos ejemplares mendocinos. Los cranidios de esa especie canadiense difieren notablemente de la especie mendocina.

Bathyuriscus terranovensis Young \& Ludvigsen, 1989, de Terranova occidental, se basa en 15 cranidios y 9 pigidios no articulados y se desconoce el tórax. Los cranidios de los especímenes canadienses se parecen a $B$. mendozanus en la forma de la glabela que es expandida hacia adelante a partir del $2^{\circ}$ surco glabelar, un elongado anillo occipital con un tubérculo medio, lóbulos palpebrales uniformemente redondeados, elongados y cuyo largo es casi la mitad del largo del cranidio, área palpebral ancha y elevada por encima de los lóbulos palpebrales, mejilla fija posterior es ancha y corta. En cambio, los pigidios de esa especie canadiense no se parecen a la especie mendocina.

Bathyuriscus aff. rotundatus (Rominger, 1887) sensu Bordonaro \& Banchig (1990: 32, lámina 1, figuras 1 y 2) de uno de los olistolitos Ojos de Agua, en la Sierra del Tontal, San Juan, se lo consideró afín con esa especie por un pigidio fragmentado con el mismo número de segmentos axiales y el borde pigidial angosto y elevado. Los cranidios se diferencian notablemente porque la glabela es menos expandida anteriormente. A partir de esta revisión se considera que tanto el cranidio como el pigidio se encuadran en el rango de variabilidad que tiene $B$. mendozanus (Fig. 5.i).

Los pigidios de Bathyuriscus sp. indet. (Bordonaro \& Banchig, 1990: 32, lámina 1, figuras 3 y 4), provenientes de otro de los olistolitos Ojos de Agua, aunque están deformados y aplastados, comparten rasgos comunes que están dentro del rango de variabilidad exhibido por $B$. mendozanus. Es destacable la cantidad de cinco anillos axiales más una pieza terminal triangular que no toca el borde pigidial y el borde pigidial que está bien definido y elevado (Fig. 5.j).

Parkaspis endecamera Rasetti, 1951 en Bordonaro \& Banchig (1995: lámina 2, figura 6) fue asignado erróneamente a tal especie porque en ese único ejemplar casi completo están borrados por erosión los surcos pleurales del pigidio y no se ha conservado, por rotura, el borde pigidial. Observado este detalle, el resto de los caracteres indica que pertenece a $B$. mendozanus (Fig. 4.a)

Bathyuriscus sp. mencionado en Bordonaro \& Banchig (1996: 475) procedente de uno de los olistolitos Los Sombreros, es también considerado dentro de la variabilidad de la especie aquí revisada. Si bien el material se encuentra muy deformado y aplastado, puede apreciarse claramente ejemplares con pigidios cuyos surcos axiales y borde pigidial (Fig. 5.d-e) y cranidios con glabelas, áreas palpebrales y fixígenas (Fig. 5.f-h), que tienen características similares a $B$. mendozanus.

Bathyuriscus sp. indet. sensu Bordonaro \& Banchig (2007: 99, figura 2.F-H) de la Formación Alojamiento de Mendoza, a pesar de su deficiente conservación y deformación, se pueden identificar como B. mendozanus por compartir los caracteres diagnósticos de los pigidios (cinco anillos axiales más una pieza terminal triangular que no toca el borde pigidial y el borde pigidial que está bien definido y elevado que se halla limitado con un notable surco del borde marginal) (Fig. 5.a-c).

Figura 5. a-c, Bathyuriscus mendozanus (Rusconi, 1945) de la Formación Alojamiento, quebrada de Aguilera, Cordón del Alojamiento, Mendoza, a, pigidio IANIGLA-PI 1579. b, pigidio IANIGLA-PI 1588. c, cranidio IANIGLA-PI 1581. d-h, $B a-$ thyuriscus mendozanus (Rusconi) de los olistolitos Los Sombreros, Formación Los Sombreros, quebrada del Salto, Sierra del Tontal, San Juan, d, pigidio IANIGLA-PI 2345. e, pigidio IANIGLA-PI 2344. f, cranidio IANIGLA-PI 2339. g, cranidio IANIGLA-PI 2340. h, cranidio IANIGLA-PI 2341. i-j, Bathyuriscus mendozanus (Rusconi) de los olistolitos Ojos de Agua, Formación Los Sombreros, quebrada de Ojos de Agua, Sierra del Tontal, San Juan. i, cranidio PIUNSJ 516. j, pigidio PIUNSJ 518. Escala gráfica $=2,5 \mathrm{~mm}$.

a-c, Bathyuriscus mendozanus (Rusconi, 1945) of the Alojamiento Forrmation, quebrada de Aguilera, Cordón del Alojamiento, Mendoza, a, pygidium IANIGLA-PI 1579. b, pygidium IANIGLA-PI 1588. c, cranidium IANIGLA-PI 1581. d-h, Bathyuriscus mendozanus (Rusconi) of the Los Sombreros olistoliths, Los Sombreros Formation, quebrada del Salto, Sierra del Tontal, San Juan, d, pygidium IANIGLA-PI 2345. e, pygidium IANIGLA-PI 2344. f, cranidium IANIGLA-PI 2339. g, cranidium IANIGLA-PI 2340. h, cranidium IANIGLA-PI 2341. i-j, Bathyuriscus mendozanus (Rusconi) of the Ojos de Agua olistoliths, Los Sombreros Formation, quebrada de Ojos de Agua, Sierra del Tontal, San Juan. i, cranidium PIUNSJ 516. $\boldsymbol{j}$, pygidium PIUNSJ 518. Bar scale $=2.5 \mathrm{~mm}$. 
Horizonte y Edad. Cámbrico Medio, Piso Marjumiense, Zona de Oryctocephalus, niveles superiores de los olistolitos San Isidro alojados en la Formación Empozada, Precordillera de Mendoza. Zona de Bathyuriscus-Elrathina de los olistolitos de Ojos de Agua y de Los Sombreros, incluidos en el tramo medio e inferior respectivamente de la Formación Los Sombreros, Precordillera occidental de San Juan. Zona de Oryctocephalus, tramo medio de la Formación Alojamiento en la quebrada de Aguilera en el Cordón del Alojamiento, Precordillera de Mendoza. Según el esquema bioestratigráfico mundial propuesto por la International Subcomission on Cambrian Stratigraphy (ISCS) se ubica en la Series 3 (antiguo Cámbrico Medio), en la parte superior del Piso 5 del Cámbrico.

\section{AGRADECIMIENTOS}

Se agradece a la Licenciada Susana Devincenzi del Museo de Ciencias Naturales y Antropológicas "Juan Cornelio Moyano" de Mendoza, por facilitar los ejemplares de la colección Rusconi para su estudio. Al Sr. Rafael Bottero, técnico del IANIGLA, por la confección de las láminas y figuras. Al Sr. Alberto Acosta, técnico de la Dirección de Recursos Naturales Renovables de Mendoza, por la colaboración en las campañas y en los hallazgos de trilobites en San Isidro. A Mark Webster por proporcionar información sobre la sinonimia de Bathyuriscus registrada en el Instituto de Estudios Cámbricos de la Universidad de Chicago. A los revisores de este trabajo, R. Gozalo y E. Liñán, por las mejoras hechas al manuscrito. El presente trabajo fue financiado por el PIP 084(2010 -1012) de CONICET de Argentina.

\section{BIBLIOGRAFÍA}

Babcock, L.E. 1994. Systematics and phylogenetics of polymeroid trilobites from the Henson Gletscher and Kap Stanton formations (Middle Cambrian), North Greenland. Gronland Geologiske Undersogelse Bulletin, 169, 79-127.

Bergström, J. \& Levi-Setti, R. 1978. Phenotypic variation in the Middle Cambrian Paradoxides davidis Salter at Manuels, SE Newfoundland. Geologica et Paleontologica, 12, 1-40.

Banchig, A.L. 2006. Formación Alojamiento (Cámbrico) en su localidad tipo. Paleoambiente sedimentario del margen continental eopaleozoico, Precordillera mendocina. Revista de la Asociación Geológica Argentina, 61, 301-312.

Banchig, A.L. \& Bordonaro, O.L. 1994. Reinterpretación de la Formación Los Sombreros: Secuencia olistostrómica de talud, Precordillera Argentina. V Reunión Argentina de Sedimentología, 283-288.

Bordonaro, O.L. 1990. El Sistema Cámbrico de la Provincia de San Juan. In: Relatorio de Geología y Recursos Naturales de la Provincia de San Juan (Ed. O.L. Bordonaro). XI Congreso Geológico Argentino, San Juan, 18-30.

Bordonaro, O.L. 2003. Evolución paleoambiental y paleogeográfica de la cuenca cámbrica de la Precordillera argentina. Revista de la Asociación Geológica Argentina, 58, 329-346.

Bordonaro, O.L. \& Banchig, A.L. 1990. Nuevos trilobites del cámbrico medio en la quebrada Ojos de Agua, Sierra del Tontal, San Juan (Argentina). V Congreso Argentino de Paleontología y Bioestratigrafía, 31-37.

Bordonaro, O.L. \& Banchig, A.L. 1995. Trilobites laurénticos en el cámbrico de la Precordillera argentina. VI Congreso Argentino de Paleontología y Bioestratigrafia, 1, 59-65.

Bordonaro, O.L. \& Banchig, A.L. 1996. Estratigrafía de los olistolitos cámbricos de la Precordillera Argentina. XIII Congreso Geológico Argentino y III Congreso de exploración de Hidrocarburos, 5, 471-479.

Bordonaro, O.L. \& Banchig, A.L. 2007. Biofacies de trilobites cámbricos en la Formación Alojamiento, Precordillera de San Juan y Mendoza, Argentina. Ameghiniana, 44, 91-107.

Bordonaro, O.L., Beresi, M.S. \& Keller, M. 1993. Reinterpretación estratigráfica del cámbrico del área de San Isidro, Precordillera de Mendoza. XII Congreso Geológico Argentino y II Congreso de Exploración de Hidrocarburos, 2, 12-19.

Bordonaro, O.L., Banchig, A.L., Pratt, B.R. \& Raviolo, M.M. 2008. Trilobite-based biostratigraphic model (biofacies and biozonation) for the Middle Cambrian carbonate platform of the Argentine Precordillera. Geologica Acta, 6, 115-129.

Bright, R.C. 1959. A paleoecologic and biometric study of the Midle Cambrian trilobite Elrathia kingii (Meek). Journal of Paleontology, 33, 83-98.

Castellaro, H.A. 1963. Guía Paleontológica Argentina, Parte 1: Paleozoico, Sección I: Faunas Cámbricas. Consejo Nacional de Investigaciones Científicas y Técnicas, 13-37.

Cuerda, A., Cingolani, C. \& Varela, R. 1983. Las graptofaunas de la Formación Los Sombreros, Ordovícico Inferior de la sierra del Tontal, Precordillera de San Juan. Ameghiniana, 20, 239-260.

Fritz, W.H. 1968. Lower and Early Middle Cambrian trilobites from the Pioche Shale, East-Central Nevada, U.S.A. Palaeontology, 11, 183-235.

Harrington, H.J. \& Leanza, A.F. 1942. Sobre algunos trilobites nuevos o poco conocidos del Ordovícico argentino. Revista del Museo de la Plata, n. s., 2, 131-141.

Hughes, N.C. 1994. Ontogeny, intraspecific variation, and systematics of the Late Cambrian trilobite Dikelocephalus. Smithsonian Contributions to Paleobiology, 79, 1-89.

Keller, M., Bordonaro, O.L. \& Beresi, M.S. 1993. The Cambrian of San Isidro, Mendoza, Argentina: Facies and sedimentology at the platform slope transition. Neues Jahrbuch fur Geologie und Palaontologie, Monatshefte, 1993 (H6), 373-383.

Kindle, C.H. 1982. The C.H. Kindle Collection: Middle Cambrian to Lower Ordovician trilobites from the Cow Head Group, Western Newfoundland. Geological Survey of Canada, 82 (1C), 1-17.

Leanza, A.F. 1947. El Cámbrico Medio de Mendoza. Revista del Museo de La Plata, Sección Paleontología, 3, 223-235.

McLaughlin K.P. \& Enbysk, B.B. 1950. Midle Cambrian trilobites from Pend Oreille County, Washington. Journal of Paleontology, 24, 466-471. 
Meek, F.B. 1873. Preliminary paleontological report, consisting of lists of fossils, with remarks on the ages of the rocks in wich they were found. United States Geological Survey territory $6^{\circ}$ Annual Report, 429-518.

Owen, D.D. 1852. Report of the Geological Survey of Wisconsin, Iowa and Minnesota. Lippencott, Grambo and Co., Philadelphia, 638 pp.

Palmer, A.R. 1968. Cambrian trilobites of East-Central Alaska. U.S. Geological Survey Professional Paper, 559-B, $115 \mathrm{pp}$.

Poulsen, V. 1958. Contributions to the Middle Cambrian Paleontology and Stratigraphy of Argentina. Matemnatisk Fysiske Meddelelser Danske Videnskabernes Selskab, 31 (8), 1-22.

Ramos, V.A. 2004. Cuyania, an exotic block to Gondwana: Review of a historical success and the present problems. Gondwana Research, 7, 1009-1026.

Rasetti, F. 1948. Middle Cambrian trilobites from the conglomerates of Quebec (exclusive of the Ptchopariidea). Journal of Paleontology, 22, 315-339.

Rasetti, F. 1951. Middle Cambrian stratigraphy and faunas of the Canadian Rocky Mountains. Smithsonian Miscellaneous Collections, 116 ( $\mathrm{n}^{\circ}$ 5), $255 \mathrm{pp}$.

Rasetti, F. 1967. Lower and Middle Cambrian trilobite faunas from the Taconic sequence of New York. Smithsonian Miscellaneous Collections, 152 (4), 112 pp.

Robison, R.A. 1964. Late Middle Cambrian faunas from the western Utah. Journal of Paleontology, 38, 510-566.

Robison, R.A. 1967. Ontogeny of Bathyuriscus fimbriatus and it bearing on affinities of Corynexochid trilobites. Journal of Paleontology, 41, 213-221.

Robison. R.A., 1971. Additional Middle Cambrian trilobites from the Wheeler Shale of Utah. Journal of Paleontology, 45, 796-804.
Robison, R.A. 1976. Middle Cambrian trilobite biostratigraphy of the Great Basin. Brigham Young University Geology Studies, 23, 93-109.

Rominger, C. 1887. Description of Primordial fossils from Mt. Stephens, North-Western territory of Canada. Proceedings Academy of Natural Sciences Philadelphia, 1887, 12-19.

Rusconi, C. 1945. Trilobites silúricos de Mendoza. Anales de la Sociedad Cientifica Argentina, 139, 216-219.

Rusconi, C. 1950. Notas sobre faunas cámbricas de Mendoza. Anales de la Sociedad Cientifica Argentina, 149, 157-177.

Rusconi, C. 1952. Fósiles cámbricos del cerro Áspero, Mendoza. Revista del Museo de Historia Natural de Mendoza, 6, 63-122.

Sundberg, F.A. 1994. Corynexochida and Ptychopariida (Trilobita, Arthropoda) of the Ehmaniella Biozone (Middle Cambrian), Utah and Nevada. Natural History Museum of Los Angeles County, Contributions in Science, 446, 137 pp.

Tortello, F.M.\&Bordonaro, O.L. 1997. Cambrianagnostoid trilobites from Mendoza,Argentina:Asystematicrevisionandbiostratigraphyc implications. Journal of Palentology, 71, 74-86.

Walcott, C.D. 1916. Cambrian Geology and Paleontology, 3, 5-Cambrian trilobites. Smithsonian Miscellaneous Collections, 64 (5), 303-456.

Wolfart, R. 1994. Middle Cambrian faunas (Brachiopoda, Mollusca, Trilobita) from the exotic limestone blocks, Reilly Ridge, North Victoria Land, Antarctica; their biostratigraphic and paleobiogeographic significance. Geologisches Jahrbuch, B84, 161 pp.

Young, G.A. \& Ludvigsen, R. 1989. Mid - Cambrian trilobites from the lowest par of the Cow Head, Western Newfoundland. Geological Survey of Canada, Bulletin, 392, 1-49.

Manuscrito recibido: 28 de abril, 2011 Manuscrito aceptado: 8 de junio, 2011 